\title{
Visual failure in allergic aspergillus sinusitis: case report
}

\author{
IAIN S DUNLOP AND FRANK A BILLSON \\ From Sydney Eye Hospital, Sydney, Australia
}

SUMMARY We present a case of rapid progressive unilateral visual loss in a 69-year-old woman who presented with facial pain, ipsilateral proptosis and restriction of eye movements, and nasal symptoms suggestive of sinusitis. A diagnosis of allergic aspergillus sinusitis was made on the basis of local histopathology and systemic features. Over a three-week period vision deteriorated to bare perception of light but showed a dramatic improvement to a level of $6 / 9$ central vision on systemic steroid therapy. The mechanism of visual failure in the context of allergic aspergillus sinusitis and the therapeutic implications are discussed.

Allergic aspergillus sinusitis has recently been described $^{2}$ and appears to be analogous to allergic bronchopulmonary aspergillosis (ABPA). This similarity has important diagnostic and therapeutic implications which differ markedly from those associated with the more familiar infective aspergillosis of the sinuses and their adnexae.

We report a case of an elderly woman with characteristic paranasal sinus involvement and right orbital complications. A relentless decrease in right visual acuity to minimal light perception was arrested by recognition of the condition and prompt appropriate treatment. She rapidly regained, and has sustained, right acuity of $6 / 9$ corrected during nine months' follow-up.

\section{Case report}

A previously fit and alert 69-year-old woman was admitted with a five-day history of headache, right periorbital and frontal pain, nausea, and progressive diminution of right visual acuity (VA) associated with right proptosis and a more recent onset of diplopia. She complained of three weeks of vague nasal congestion but denied nasal discharge.

Her general health had been good. She denied any history of asthma and/or chronic or recurrent respiratory symptoms. She denied atopy and significant allergies. She was a non-smoker and took no regular medication.

Correspondence to Professor F A Billson, Sydney Eye Hospital, Sir John Young Crescent, Woolloomooloo, NSW 2011, Australia.
Examination on day one (D1) showed the right VA was approximately $6 / 24$, the left $6 / 9$. There was mild right periorbital swelling and proptosis, but little chemosis. She had a right relative afferent pupillary defect. Abduction and vertical deviations in abduction were moderately impaired on the right. The fundi were normal; in particular the right disc was not swollen or haemorrhagic, nor were the right retinal veins engorged.

The patient was afebrile, with leucocytes $7 \cdot 9 \times 10^{9} / 1$, and erythrocyte sedimentation rate $32 \mathrm{~mm}$ in the first hour. The chest $x$-ray was normal. Skull $x$-rays showed clouded ethmoidal and frontal sinuses. Cranial CT confirmed soft tissue density in the ethmoidal sinuses and fluid in the sphenoid sinus, and right proptosis associated with increases in the orbital soft tissue density. There was no specific abnormality of the optic nerves nor of the optic canals.

On D2 an external ethmoidectomy and sphenoidotomy was performed via the right medial orbital wall. A large volume of mucoid granulation tissue was removed from the ethmoid and sphenoidal sinuses. Gram staining revealed no organisms. Pus cells were abundant, but culture failed to produce any significant growth. Further fungal studies were undertaken and later produced no growth.

Treatment continued with flucloxacillin $1 \mathrm{~g} 4$-hourly intravenously, amoxycillin 1 $\mathrm{g}$ 4-hourly intravenously, gentamicin $60 \mathrm{mg}$ 6-hourly intravenously, and neosynephrine nasal spray 6-hourly.

Postoperatively her condition failed to improve. On D8 the right VA was minimal light perception 


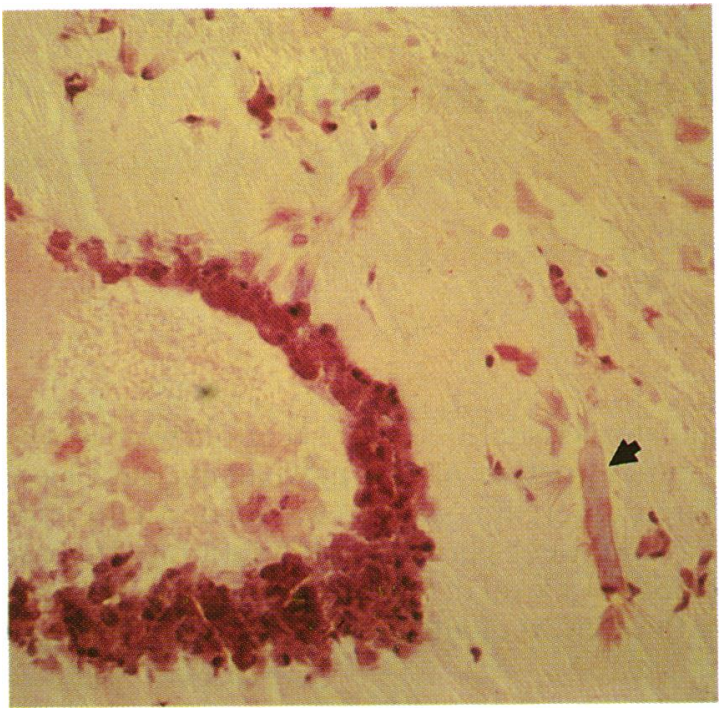

Fig. 1 High-power view of paranasal drainage specimen showing a characteristic strip of inspissated eosinophils and a large Charcot-Leyden crystal (arrowed) in a mucinous background. Haematoxylin-eosin.

with an absolute afferent pupil defect. The proptosis $(19 \mathrm{~mm} / \mathrm{R}, 14 \mathrm{~mm} / \mathrm{L})$ and mild periorbital oedema persisted. The right eye was exotropic and hypertropic with minimal ductions. The fundal findings remained normal.

A repeated cranial CT on that day revealed large amounts of fluid in maxillary, ethmoid, and sphenoid sinuses, with signs of mucosal thickening. The operative loss of the right lateral ethmoidal wall was noted. The right medial rectus was laterally displaced, and increases in orbital soft tissue density persisted. A differential full blood count showed leucocytes $6.7 \times 10^{9} / 1$ with $9 \%$ eosinophils.

Antibiotic therapy was changed to cephalothin $1 \mathrm{~g}$ 4-hourly intravenously and metronidazole $200 \mathrm{mg}$ 6-hourly by mouth. Again there was no significant clinical improvement.

On D12 histopathology suggested a diagnosis of allergic aspergillus sinusitis. The operative specimens revealed mucous masses surrounded by respiratory mucosa. In the mucous were Charcot-Leyden crystals associated with clusters and long strips of inspissated eosinophils. These clusters were sharply angulated, suggesting that they had broken off from a larger mass. Although no fungal filaments were demonstrated, the appearance was characteristic of the changes found in allergic bronchopulmonary aspergillosis $^{3}$ and allergic aspergillus sinusitis. ${ }^{12}$

Antibiotics were stopped and prednisone $15 \mathrm{mg}$ twice daily by mouth was begun on D13. Metronida-

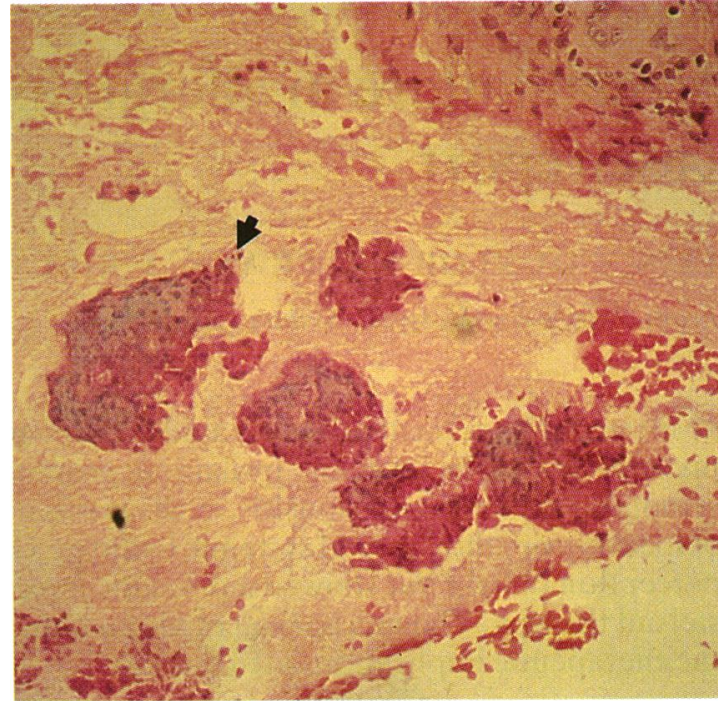

Fig. 2 High-power view of paranasal drainage specimen showing an angulated cluster of eosinophils (arrowed) suggestive of fragmentation of a larger mass. Haematoxylineosin.

zole continued until D15. Despite corticosteroid therapy, Aspergillus fumigatus serum precipitins were requested and returned negative results at both high $(20 \mathrm{~g} / \mathrm{l})$ and low $(2 \mathrm{~g} / \mathrm{l})$ concentrations. Serum IgE levels were not measured.

The patient noted an immediate improvement. On D18, she could count fingers poorly and had developed a right relative afferent pupil defect. Her right ductions improved and right exotropia decreased. On D22 the right VA was $6 / 18$ corrected. The afferent defect had resolved. Ductions were only mildly impaired and the right visual field (Bjerrum) was normal. Prednisone was decreased to $12.5 \mathrm{mg}$ twice daily. On D32 the right VA was $6 / 12$ corrected. This further improved to $6 / 9$ corrected by D41 and has remained stable since.

Checkerboard visual evoked responses performed on D41 showed a normal left response but increased latency (+122 ms) and decreased amplitude on the right consistent with a persistent right optic neuropathy.

At 12 months she was currently asymptomatic and on prednisone $5 \mathrm{mg}$ twice daily.

\section{Discussion}

Unilateral visual loss in the elderly is most often acute and irreversible, owing to vascular events in the field of the ophthalmic artery. Progressive visual loss with pain and proptosis more often arises from optic nerve 
involvement in orbital cellulitis, either primary or secondary to paranasal sinus infection, which may be due to a multiplicity of causative organisms including the potentially lethal mucormycoses associated with diabetes mellitus. Tumour, either primary or secondary of the orbit, paranasal sinuses, nasopharynx, or parasellar regions may also present in this way, though pain is less often a feature-pain occurring more frequently in pseudotumour.

Careful history taking and radiological investigations including CT scanning will usually provide evidence for the diagnosis. In the present case the history was not suggestive of a vascular cause, and the pattern of involvement of multiple sinuses made malignancy unlikely.

In the context of paranasal sinusitis impaired vision is normally an indication for surgical drainage, at which time both microbiological and histopathological specimens may be obtained. Aeration of the ethmoids associated with pneumatisation of the optic canal or sphenoidal mucocele involving the optic canal can usually be demonstrated by careful radiological examination. Where there is no bony involvement in the presence of a systemic eosinophilia, the diagnosis of allergic aspergillus sinusitis should be considered. In the past, visual failure has not been regarded as a serious threat in allergic aspergillus sinusitis, though this case illustrates that the diagnosis should be considered when culture of organisms is negative and a visual deficit progresses despite surgical drainage of the paranasal sinuses.

Allergic aspergillus sinusitis ${ }^{12}$ is a very different entity to the uncommon but more familiar infective aspergillosis of the paranasal sinuses and/or orbit. Chronic sinusitis is mainly due to aerobic and anaerobic bacterial infection. When, infrequently, true fungal infection occurs, it has been subdivided into invasive and non-invasive forms.

The invasive form has a spectrum of activity from a slow and relatively asymptomatic form to a fulminant one seen in the debilitated and immunosuppressed, where $60 \%$ mortality has been reported. ${ }^{45}$ Single sinus involvement occurs in more than half these cases. ${ }^{124}$ Histologically there is marked tissue necrosis with granulomatous and fibrotic reactions associated with abundant characteristic fungal hyphae. The non-invasive form is equivalent to the pulmonary mycetoma. It usually involves a single sinus, presenting a dense tangled mass of hyphae associated with little inflammatory reaction. The treatment of both types involves local surgical excision and antifungal agents. In each case hyphae are numerous; in neither is mucin production a major feature.

Allergic aspergillus sinusitis has recently been described as a new and distinct clinicopathological entity analogous to allergic bronchopulmonary aspergillosis (ABPA). ${ }^{12}$ The latter has long been recognised as an aspergillus hypersensitivity manifestation of the bronchopulmonary tree with specific histopathological features and treatment implications. Allergic aspergillus sinusitis, occurring in the paranasal sinuses without, necessarily, concomitant lung involvement, shares these features-opacification of multiple sinuses with distinct mucinous material containing eosinophils, Charcot-Leyden crystals, and fungal hyphae.' This material is characterised by aggregation of necrotic inspissated eosinophils lying in thin strips or clusters that frequently show angulation suggestive of cleavage from a larger mass. ${ }^{3}$ The background mucous contains nuclear debris, sloughed respiratory mucosal cells, free eosinophil granules, and Charcot-Leyden crystals. These crystals, hexagonal in cross section, bipyramidal in longitudinal section, and of variable size, form from aggregation of eosinophil granules. ${ }^{2}$ This constellation of findings is indicative of 'allergic mucin'.

On histological grounds and in terms of response to treatment we believe this to be such a case despite certain clinical differences. In Katzenstein's retrospective series, age range $13-48$, most patients were young adults with a history of asthma and chronic sinus involvement. All had multiple sinus involvement, and most a normal chest $x$-ray. Several had undergone recurrent surgical drainage procedures. Our patient is elderly. She denies chronic nasal or respiratory problems and related surgery. However, it is noteworthy that she presented late, with primarily ocular symptoms. Her failure to respond to surgical drainage and intensive antibiotic therapy, worrisome at the time, is typical of the condition.

The diagnosis was made on histopathological appearances. Culture of operative specimens was unhelpful. A tell-tale eosinophilia within a normal white cell count was evident when sought. 'Allergic mucin" 2 was abundant in the specimens. Jelihovsky ${ }^{3}$ reports its most distinctive feature as inspissated, often angular, clusters of eosinophils, within some of which fungal hyphae are found. In one reported case, submitted to 10 drainage procedures, allergic mucin was found on three occasions, only one of which contained identifiable hyphae. As the hyphae are not numerous, unlike infective aspergillosis, sampling difficulty may be the explanation. It is also possible that an allergen other than aspergillus is present.'

The mechanism of visual loss in this case is not certain, and we have been unable to find reports of such loss in this condition. It may be that the orbital inflammatory changes, sufficient to cause proptosis and minimal ductions, compress the optic nerve 
proximally to produce an 'orbital apex syndrome', such as that seen in dysthyroid optic neuropathy. However, the recti, with the exception of the medial rectus, were not grossly enlarged on CT. It is also possible that the right optic nerve was directly involved in the orbital and paranasal inflammation. The latter possibility is in keeping with the impressive response to steroids. It is also more likely on the basis of the increased latency and diminished amplitude of the checkerboard VEP.

There are no prospective data on optimal therapy for allergic aspergillus sinusitis. The remarkable and sustained recovery in this case reinforces the primary role of corticosteroids. This is consistent with Safirstein's report of a young woman with proved ABPA and chronic sinusitis now presumed to be allergic aspergillus sinusitis. ${ }^{6}$ The sinus-related histopathology was not described. She experienced a 'dramatic' and simultaneous improvement of lung and nasal conditions on prednisone $(30 \mathrm{mg})$, and exacerbations thereof on cessation. These results with corticosteroids are in sharp contrast to those achieved with surgical drainage and antifungal agents, where multiple recurrences are common.

The role of serology in diagnosis is not clear. Our negative results to Aspergillus fumigatus precipitins were complicated by concomitant steroid therapy. Serum IgE was not tested. Both these tests are useful but not absolute in ABPA and were positive in the majority of those tested in Katzenstein and colleagues' series.' It is clear that relevant peripheral blood studies, differential leucocyte counts for eosinophilia, and tests for IgE and precipitins, should be done early in investigation of these patients.

In conclusion, we present what we believe to be a case of allergic aspergillus sinusitis with right orbital complications and progressive visual loss. The patient's age and previous respiratory history are atypical. Her poor response to surgical drainage is typical. The histopathological features of 'allergic mucin' are characteristic, while the absence of identifiable fungal hyphae is not unusual. The peripheral eosinophilia should have been recognised earlier. Most importantly, there was an immediate and sustained recovery from blindness to comfortable reading vision with corticosteroid therapy.

With sophisticated radiological imaging to confirm the diagnosis in the presence of a systemic eosinophilia and positive serology, a presumptive diagnosis of allergic aspergillus sinusitis followed by a trial of steroid therapy without formal surgical exploration is, we believe, a reasonable approach to management. Should doubt exist, surgical intervention provides both appropriate drainage for true paranasal sinus infection and microbiological and histological specimens for pathological diagnosis.

Allergic aspergillus sinusitis is a newly recognised and uncommon condition. Its association with reversible visual loss is important. In our present state of knowledge nature seems capricious in choosing to affect certain individuals. While prednisone appears to offer dramatic improvement, so too may steroids initially attenuate ocular and paranasal tumours. We believe that allergic aspergillus sinusitis warrants a wider press and higher index of suspicion. Further information on optimal therapy and longterm follow up would be helpful.

Note ADDED IN PROOF. At 22 months follow-up central acuity remains $6 / 9$ with no indication of visual deterioration on therapy with prednisone $2 \mathrm{mg}$ on alternate days.

We thank Dr Tatiana Jelihovsky for her histopathological diagnosis and relevant photographs.

\section{References}

1 Katzenstein A, Sale S, Greenberger P. Allergic aspergillus sinusitis: a newly recognised form of sinusitis. J Allergy Clin Immunol 1983; 72: 89-93.

2 Katzenstein A, Sale S, Greenberger P. Pathologic findings in allergic aspergillus sinusitis. Am J Surg Pathol 1983; 7: 439-43.

3 Jelihovsky T. The structure of bronchial plugs in mucoid impaction, bronchocentric granulomatosis and asthma. Histopathology 1983; 7: 153-67.

4 Green WR, Font RL, Zimmerman LE. Aspergillosis of the orbit. Arch Ophthalmol 1969; 82: 302-13.

5 Wolter JR. Diagnosis and management of orbital aspergillosis. Ann Ophthalmol 1976; 8: 17-20.

6 Safirstein B. Allergic bronchopulmonary aspergillosis with obstruction of the upper respiratory tract. Chest 1976; 70: 788-90.

Accepted for publication 15 December 1986. 